\title{
NY-BR-1 protein expression in breast carcinoma: a mammary gland differentiation antigen as target for cancer immunotherapy
}

Theurillat, J P ; Zürrer-Härdi, U ; Varga, Z ; Storz, M ; Probst-Hensch, N M ; Seifert, Burkhardt ; Fehr, M K ; Fink, D ; Ferrone, S ; Pestalozzi, B ; Jungbluth, A A ; Chen, Y T ; Jäger, D ; Knuth, A ; Moch, H

\begin{abstract}
NY-BR-1 is a recently identified differentiation antigen of the mammary gland. To use NYBR-1 for T-cell-based immunotherapy, analysis of its co-expression with HLA class I antigens is required. In the present tissue microarray study, primary breast cancers $(\mathrm{n}=1,444)$, recurrences $(\mathrm{n}=88)$, lymph node $(\mathrm{n}=525)$ and distant metastases $(\mathrm{n}=91)$ were studied for NY-BR-1 expression using a novel monoclonal antibody. NY-BR-1 expression was compared with prognosis, estrogen receptor, HER2status, EGFR and HLA class I antigen expression. NY-BR-1 was more frequently expressed in grade 1 $(82 \%)$ than in grade $2(69 \%)$ and grade $3(46 \%)$ carcinomas $(\mathrm{P}<0.0001)$. Moreover, NY-BR-1 expression correlated directly with estrogen receptor expression $(\mathrm{P}<0.0001)$ and inversely correlated with HER2status and EGFR expression $(\mathrm{P}<0.0001$ for both). Considering high expression level of co-expression, 198/1,321 (15\%) primary breast carcinomas and 4/65 (6\%) distant metastases expressed NY-BR-1 and HLA class I, suggesting that active immunotherapy can be applied to about $10 \%$ of breast cancer patients. Survival analysis showed an association of NY-BR-1 expression with better patient outcome $(\mathrm{P}=0.015)$. No difference between NY-BR-1 expression of primary tumors and metastases could be found, indicating that the presence of NY-BR-1 in metastases can be deduced from their corresponding primary. Forty-three paired biopsies taken from patients before and after chemotherapy suggest that NY-BR-1 expression is not influenced by preceding chemotherapy (kappa $=0.89, \mathrm{P}<0.0001)$. In summary, the co-expression of NYBR-1 with HLA class I antigens and its expression in metastases without modification by chemotherapy suggest that NY-BR-1 targeted immunotherapy represents a viable strategy in addition to other targeted cancer drug therapies of breast cancer.
\end{abstract}

DOI: https://doi.org/10.1007/s00262-007-0316-1

Posted at the Zurich Open Repository and Archive, University of Zurich ZORA URL: https://doi.org/10.5167/uzh-18090

Journal Article

Published Version

Originally published at:

Theurillat, J P; Zürrer-Härdi, U; Varga, Z; Storz, M; Probst-Hensch, N M; Seifert, Burkhardt; Fehr, M K; Fink, D; Ferrone, S; Pestalozzi, B; Jungbluth, A A; Chen, Y T; Jäger, D; Knuth, A; Moch, H (2007). NY-BR-1 protein expression in breast carcinoma: a mammary gland differentiation antigen as target for cancer immunotherapy. Cancer Immunology, Immunotherapy, 56(11):1723-1731.

DOI: https://doi.org/10.1007/s00262-007-0316-1 


\title{
NY-BR-1 protein expression in breast carcinoma: a mammary gland differentiation antigen as target for cancer immunotherapy
}

\author{
Jean-Philippe Theurillat • Ursina Zürrer-Härdi · Zsuzsanna Varga • Martina Storz • Nicole M. Probst-Hensch • \\ Burkhardt Seifert · Mathias K. Fehr · Daniel Fink - Soldano Ferrone $\cdot$ Bernhard Pestalozzi $\cdot$ \\ Achim A. Jungbluth · Yao-Tseng Chen · Dirk Jäger • Alexander Knuth · Holger Moch
}

Received: 17 November 2006 / Accepted: 10 March 2007 / Published online: 5 April 2007

(c) Springer-Verlag 2007

\begin{abstract}
NY-BR-1 is a recently identified differentiation antigen of the mammary gland. To use NY-BR-1 for Tcell-based immunotherapy, analysis of its co-expression with HLA class I antigens is required. In the present tissue microarray study, primary breast cancers $(n=1,444)$, recurrences $(n=88)$, lymph node $(n=525)$ and distant metastases $(n=91)$ were studied for NY-BR-1 expression using a novel monoclonal antibody. NY-BR-1 expression was compared with prognosis, estrogen receptor, HER2-status, EGFR and HLA class I antigen expression. NY-BR-1 was more frequently expressed in grade $1(82 \%)$ than in grade 2 $(69 \%)$ and grade $3(46 \%)$ carcinomas $(P<0.0001)$. Moreover, NY-BR-1 expression correlated directly with estrogen
\end{abstract}

Jean-Philippe Theurillat and Ursina Zürrer-Härdi contributed equally to this study.

J.-P. Theurillat $(\bowtie) \cdot$ U. Zürrer-Härdi $\cdot$ Z. Varga $\cdot$

M. Storz $\cdot$ H. Moch

Institute for Surgical Pathology,

Department of Pathology, University Hospital Zurich,

Schmelzbergstrasse 12, 8091 Zurich, Switzerland

e-mail: Jean-Philippe.Theurillat@cell.biol.ethz.ch

N. M. Probst-Hensch

Molecular Epidemiology Unit/Cancer Registry Zürich,

University Hospital of Zurich, Zurich, Switzerland

B. Pestalozzi · D. Jäger · A. Knuth

Clinic of Oncology, Department of Internal Medicine,

University Hospital of Zurich, Zurich, Switzerland

M. K. Fehr · D. Fink

Department of Obstetrics and Gynecology,

University Hospital of Zurich, Zurich, Switzerland

B. Seifert

Division of Biostatistics, University Zurich,

Zurich, Switzerland receptor expression $(P<0.0001)$ and inversely correlated with HER2-status and EGFR expression $(P<0.0001$ for both). Considering high expression level of co-expression, 198/1,321 (15\%) primary breast carcinomas and 4/65 (6\%) distant metastases expressed NY-BR-1 and HLA class I, suggesting that active immunotherapy can be applied to about $10 \%$ of breast cancer patients. Survival analysis showed an association of NY-BR-1 expression with better patient outcome $(P=0.015)$. No difference between NYBR-1 expression of primary tumors and metastases could be found, indicating that the presence of NY-BR-1 in metastases can be deduced from their corresponding primary. Forty-three paired biopsies taken from patients before and after chemotherapy suggest that NY-BR-1 expression is not influenced by preceding chemotherapy ( $\kappa=0.89, P<0.0001)$. In summary, the co-expression of

\author{
S. Ferrone \\ Department of Immunology, \\ Roswell Park Cancer Institute, \\ Buffalo, NY, USA \\ A. A. Jungbluth \\ Ludwig Institute for Cancer Research, \\ New York, USA \\ Y.-T. Chen \\ Department of Pathology, \\ Weill Medical College of Cornell University, \\ New York, USA
}

Present Address:

D. Jäger

Medical Oncology,

National Center for Tumor Diseases,

University Hospital Heidelberg,

Heidelberg, Germany 
NY-BR-1 with HLA class I antigens and its expression in metastases without modification by chemotherapy suggest that NY-BR-1 targeted immunotherapy represents a viable strategy in addition to other targeted cancer drug therapies of breast cancer.

Keywords NY-BR-1 · Breast cancer · Immunotherapy · Immunohistochemistry · Tissue microarray $\cdot$ HLA class I

\section{Introduction}

Targeted cancer drugs, e.g., trastuzumab against HER2 or gefitinib against EGFR, have been proven to be a novel effective strategy for cancer treatment [1,2]. T-cell-based immunotherapy targeting antigens such as cancer testis (CT) antigens or differentiation antigens is another promising new treatment modality, which is currently being tested in clinical trials [3-7]. Both targeted cancer drugs and Tcell-based immunotherapy display target specificity against cancer cells, thus reducing undesired effects as encountered with conventional cancer chemotherapy. The expression of an optimal target for tumor immunotherapy should be restricted to or at least selective in malignant lesions. Examples are $\mathrm{CT}$ antigens or differentiation antigens that remain stable during disease progression [8].

Recently, we have identified the NY-BR-1 gene by SEREX analysis (serological analysis of recombinant expression libraries) of a patient with metastatic breast carcinoma and we have shown that about $10 \%$ of the patients with NY-BR-1 positive tumors exhibited a humoral immune response to NY-BR-1 [8, 9]. In addition, others and we recently identified two HLA-A2 restricted epitopes that are recognized by CD8 positive T-cells $[10,11]$. Both epitopes are naturally processed and presented. The NYBR-1 gene is located on chromosome 10p11-p12 and is composed of 37 exons. It encodes a peptide of $\mathrm{Mr} 150,000$ 160,000 , a putative transcription factor. Since its protein expression is restricted to normal and neoplastic breast epithelium, NY-BR-1 is classified as a differentiation antigen of the mammary gland. A recent immunohistochemical study with a limited number of breast carcinoma lesions has shown that NY-BR-1 is expressed in about $60 \%$ of breast carcinoma and has confirmed the breast epithelium specificity [12]. Due to this organ-specificity, NY-BR-1 represents an attractive target for cancer immunotherapy and can be potentially used for diagnostic purposes in surgical pathology.

To explore NY-BR-1 as a potential target for cancer immunotherapy of breast cancer, more knowledge about the NY-BR-1 expression on a protein level in a larger cohort of primary tumors and distant metastases is paramount. Also, potential associations with clinical parameters and other markers such as estrogen receptor (ER) and progesterone receptor (PR) are necessary. Furthermore, for T-cell mediated immunization strategies, major histocompatibility complex (HLA) class I is required for presentation of target antigens, mediating the recognition of tumor cells by cytotoxic T-cells. Therefore, data about the presence of HLA class I and NY-BR-1 co-expression is crucial $[13,14]$.

Consequently, in the present study we determined the protein expression of NY-BR-1 in a large number of primary breast carcinomas, lymph node and distant metastases by immunohistochemistry using tissue microarrays (TMA). NY-BR-1 expression was correlated with prognosis, predictive parameters, e.g., ER, epidermal growth factor receptor (EGFR) and HER2-gene amplification as well as with HLA class I expression. Furthermore, we also analyzed the impact of chemotherapy on NY-BR-1 expression.

\section{Materials and methods}

\section{Patients and tissues}

Formalin-fixed paraffin-embedded tissue samples from patients of the University Hospital Zurich between 1991 and 2005 were retrieved from the archives of the Institute of Surgical Pathology. This project was approved by the local Commission of Ethics (ref. no. StV 12-2005). The mean age of the patients at diagnosis was 60.4 years (range 15-98). Twenty-five percent of the patients were pre-menopausal and $75 \%$ post-menopausal. H\&E stained sections of all tumors were re-evaluated by one pathologist (J.P.T.) for suitability for TMA construction. The construction of TMA has been previously described [15]. The following tissues were arrayed into seven paraffin TMAs: 144 normal breast tissues, 148 carcinoma in situ lesions, 1,444 primary invasive carcinomas, 88 local recurrences, 525 lymph node metastases and 91 distant metastases. The primary carcinomas consisted of $1,135(78.6 \%)$ invasive ductal and 217 (15.0\%) invasive lobular carcinomas, according to the WHO tumor classification. Other tumor types (6.4\%) included invasive tubular, cribriforme, medullary, mucinous, apocrine, metaplastic, glycogen-rich clear cell, micropapillary and papillary carcinomas.

According to TNM criteria 706 tumors were pT1, 685 pT2, 105 pT3 and 150 pT4. A total of 414 tumors were pN0, $604 \mathrm{pN} 1$ and $168 \mathrm{pN} 2 / \mathrm{pN} 3$. The pT-category and the pN-category could not be established for 12 and 427 cases, respectively. Histopathological grading was performed according to the modified Bloom and Richardson system [16]. Two hundred and four primary tumors were grade 1 (82\%), 708 grade 2 (69\%) and 490 grade $3(46 \%)$. 
Overall survival data from 1,091 patients were provided from the Cancer Register, Zurich. Additional data were obtained from the Department of Gynecology and the Department of Oncology of the University Hospital, Zurich.

To determine the impact of chemotherapy on NY-BR-1 expression, large paraffin sections from a previously described cohort of 43 patients were analyzed [17]. Tumor tissue before and 6 months after neoadjuvant chemotherapy was investigated for NY-BR-1 expression in each of these patients.

\section{Immunohistochemistry}

For initial analysis, immunostaining of monoclonal antibody (mAb) NY-BR-1 was adjusted to the Ventana Benchmark system by performing titrations and initial reactivity assessment in a small series of invasive breast cancer samples as previously described [12].

ER and EGFR expression was detected using the Ventana Benchmark (Ventana Medical Systems, Tucson, AZ, USA) automated stainer according to the manufacturer's recommendations with mAbs $6 \mathrm{~F} 11$ (Ventana) against estrogen receptor and mAbs 3C6 (Ventana) against EGFR.

For the analysis of HLA class I expression mAb HC10 (1:100) was used, which recognizes a determinant expressed on virtually all $B 2 \mathrm{~m}$-free HLA-B heavy chains and on B2m-free HLA-A10, HLA-A28, HLA-A29, HLAA30, HLA-A31, HLA-A32, and HLA-A33 heavy chains [18].

TMAs were analyzed with the Ventana Benchmark automated staining system (Ventana) using Ventana reagents for the entire procedure. For antigen retrieval, slides were heated with cell conditioning solution $(\mathrm{CC} 1)$ in a standard protocol. Endogenous peroxidase was blocked using the Ventana endogenous peroxidase blocking kit.

The immunohistochemical reactivity for all antibodies was measured as percentage of positive cells per spot $(0,5$, $10,20 \%$, etc.), regardless of the intensity of staining. Apart from survival analysis and examination of NY-BR-1 expression before and after chemotherapy, all statistical procedures were based on percentage of positive cells per spot. For multivariate analysis, staining was categorized into positive ( $\geq 5 \%$ positive cells) and negative $(<5 \%$ positive cells). For survival curves, kappa analysis and tables we used the following simplified scoring system for NYBR-1: negative ( $0 \%$ positive cells), weak (1-70\%), strong $(>70 \%)$. ER and EGFR stains were considered as positive when at least $5 \%$ of the tumor cells or more were positive. The expression of HLA class I was scored in three groups: negative (0-25\%), heterogeneous $(26-75 \%)$ and positive ( $>75 \%$ positive tumor cells), as it has been recently described [19].
The status of the HER2-neu proto-oncogene was assessed on TMAs by fluorescence in situ hybridization (FISH) using direct fluorescent-labeled DNA probes obtained from Pathvysion (VYSIS, Abott AG Diagnostic Division, Baar, Switzerland) and an Olympus microscope. The interpretation of the fluorescence signals was carried out according to the manufacturer's recommendations. Presence of five or more HER2-neu copy number signals was defined as amplification.

\section{Statistics}

Categorical data were compared using chi-square test. The Spearman rank correlation was used to correlate continuous (NY-BR-1, ER, EGFR and HLA class I immunohistochemistry) as well as ordinal clinicopathological parameters (stage and grade). Differences of continuous parameters (NY-BR-1) between groups (menopausal and HER2-status) were analyzed using the Mann-Whitney test. To determine, expression differences within the same patient, Wilcoxon signed rank test was used. A measure of agreement $(\kappa$ analysis) was done to analyze the congruence of NY-BR-1 expression before and after neoadjuvant chemotherapy.

Overall survival was calculated using Kaplan-Meier curves with assessment of statistical significance using logrank test. Univariate and stepwise (backwards) Cox proportional-hazard regression was used to determine hazard ratios and independent risk factors. Results were considered statistically significant if $P$ values were 0.05 or less.

\section{Results}

NY-BR-1 protein expression in primary breast carcinoma lesions

Figure 1 shows representative positive and negative immunohistochemical stainings for NY-BR-1, EGFR, ER and HLA class I. In our study we found NY-BR-1 expressed in $63.5 \%$ of all primary invasive carcinomas (Table 1). NYBR-1 was expressed in $80.7 \%$ of ductal carcinoma in situ (DCIS) and lobular carcinoma in situ (LCIS). The incidence of NY-BR-1 expression was significantly higher in lobular lesions (LCIS 100\%; invasive lobular cancer $73.8 \%$, respectively) than in ductal lesions (DCIS 78.6\%; invasive ductal cancer $61.1 \%$, respectively; $P=0.0006$ ). A heterogeneous weak to strong NY-BR-1 staining was observed in the epithelium of normal breast specimens, as described in our previous study [12]. A similar expression pattern was also present on all large tissue sections of lactating breast $(n=10)$.

Table 2 gives a summary of the association of NY-BR-1 protein expression with established prognostic factors. 
Fig. 1 Differential expression of $N Y-B R-1$, estrogen receptor (ER), EGFR and HLA class I heavy chain in breast carcinoma lesions by immunohistochemistry using mAbs. The staining for NY-BR-1 was nuclear/cytoplasmic, for ER nuclear, for EGFR membraneous/cytoplasmic and for HLA class I predominantly membranous. Only stromal and inflammatory cells were stained in negative tumor lesions
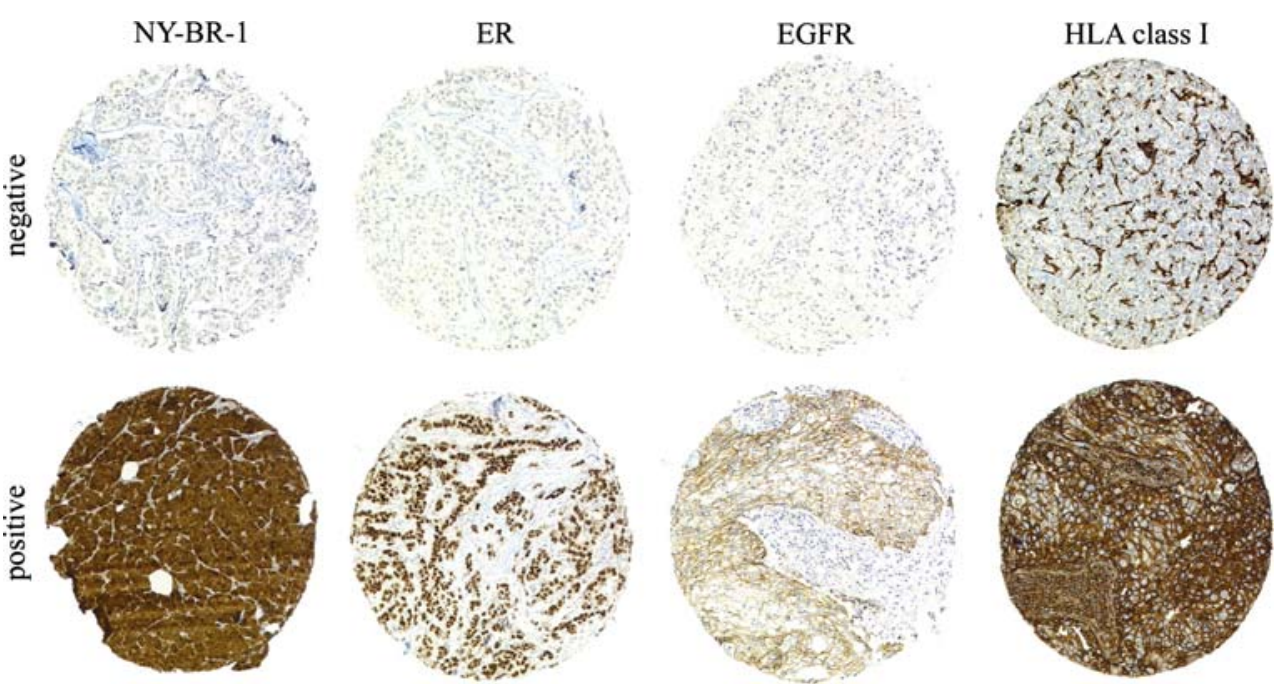

\begin{tabular}{|c|c|c|c|c|c|c|c|}
\hline \multicolumn{8}{|l|}{ NY-BR-1 expression } \\
\hline \multirow[t]{2}{*}{ Tissue type } & \multirow[b]{2}{*}{$n$} & \multicolumn{2}{|c|}{ Negative } & \multicolumn{2}{|c|}{ Weakly positive } & \multicolumn{2}{|c|}{ Strongly positive } \\
\hline & & $n$ & Percent & $n$ & Percent & $n$ & Percent \\
\hline Normal tissue & 68 & 6 & 8.8 & 54 & 79.4 & 8 & 11.8 \\
\hline \multicolumn{8}{|l|}{ In situ lesions } \\
\hline All & 145 & 28 & 19.3 & 31 & 21.4 & 86 & 59.3 \\
\hline DCIS high grade & 44 & 12 & 27.3 & 9 & 20.5 & 23 & 52.3 \\
\hline DCIS non high grade & 87 & 16 & 18.4 & 18 & 20.7 & 53 & 60.9 \\
\hline LCIS & 14 & 0 & 0 & 4 & 28.6 & 10 & 71.4 \\
\hline \multicolumn{8}{|l|}{ Invasive cancer } \\
\hline All & 1,367 & 499 & 36.5 & 335 & 24.5 & 533 & 39 \\
\hline Ductal & 1,085 & 421 & 38.8 & 249 & 22.9 & 415 & 38.2 \\
\hline Lobular & 195 & 51 & 26.2 & 65 & 33.3 & 79 & 40.5 \\
\hline Others & 86 & 27 & 31.4 & 21 & 24.4 & 38 & 44.2 \\
\hline Recurrences & 79 & 40 & 50.6 & 21 & 26.6 & 18 & 22.8 \\
\hline Lymph node metastases & 490 & 195 & 39.8 & 105 & 21.4 & 190 & 38.8 \\
\hline Distant metastases & 67 & 32 & 47.8 & 15 & 22.4 & 20 & 29.9 \\
\hline
\end{tabular}

Table 1 NY-BR-1 expression in breast lesions (the numbers in the tables are related to the number of interpretable spots on the tissue microarray)
Differences in number of cases among immunohistochemical markers were due to tissue damage (either tissue loss or inadequate tumor tissue) a problem associated with TMAs. NY-BR-1 protein expression was significantly associated with tumor grade $(P<0.0001)$, but was not associated with tumor stage (pT- and $\mathrm{pN}$-category) or bilaterality/multifocality (data not shown).

Apart from tumor grade, NY-BR-1 expression correlated significantly with ER expression $(P<0.0001)$ : $70.1 \%$ of the ER-positive tumors also co-expressed NY-BR-1, whereas over $66 \%$ of the ER-negative tumors were also negative for NY-BR-1.

NY-BR-1 expression inversely correlated to both $H E R 2$ amplification and EGFR expression $(P<0.0001$ each; Table 2), since NY-BR-1 was expressed in $65 \%$ of tumors without HER2 amplification and in $67.4 \%$ of EGFR negative tumors.

Since HLA class I molecule expression on tumor cells is crucial for their recognition by $\mathrm{T}$-cells, we screened the breast carcinoma lesions for NY-BR-1/HLA class I coexpression. Table 3 gives a summary of the association of NY-BR-1 protein expression and HLA class I expression. Among primary breast carcinomas, 587 out of 1,321 (44\%) expressed at least heterogeneously HLA class I and weakly NY-BR-1. A similar expression level of both antigens was present in 163/470 (35\%) lymph node metastases, 20/78 (25\%) recurrences, and in 17/65 (26\%) distant metastases. Corresponding strong expression levels of NY-BR-1 and HLA class I (both more than $70 \%$ of the tumor cells) was present in 198/1,321 (15\%) primaries, 45/470 (10\%) lymph 
Table 2 NY-BR-1 expression in primary invasive breast cancer according to clinicopathological variables
$S N$ sentinel (if applicable)

$N S$ not significant

${ }^{a}$ Differences in the number of cases among immunohistochemical markers was due to tissue damage (either tissue loss or inadequate tumor tissue) a problem associated with tissue microarrays

b Spearman rank correlation

${ }^{c}$ Mann-Whitney test

\begin{tabular}{|c|c|c|c|c|c|c|c|c|}
\hline \multicolumn{9}{|l|}{ NY-BR-1 expression } \\
\hline & \multirow[t]{2}{*}{$n^{\mathrm{a}}$} & \multicolumn{2}{|c|}{ Negative } & \multicolumn{2}{|c|}{ Weakly positive } & \multicolumn{2}{|c|}{ Strongly positive } & \multirow[t]{2}{*}{$P$ value } \\
\hline & & $n$ & Percent & $n$ & Percent & $n$ & Percent & \\
\hline \multicolumn{9}{|l|}{ Histologic grade } \\
\hline G1 (well differentiated) & 210 & 36 & 17.1 & 65 & 31.0 & 109 & 51.9 & $<0.0001^{\mathrm{b}}$ \\
\hline G2 (moderately differentiated) & 667 & 202 & 30.3 & 171 & 25.6 & 294 & 44.1 & \\
\hline G3 (poorly differentiated) & 469 & 256 & 54.6 & 92 & 19.6 & 121 & 25.8 & \\
\hline \multicolumn{9}{|l|}{ pT-category } \\
\hline pT1 & 535 & 165 & 30.8 & 151 & 28.2 & 219 & 40.9 & $\mathrm{NS}^{\mathrm{b}}$ \\
\hline pT2 & 602 & 238 & 39.5 & 124 & 20.6 & 240 & 39.9 & \\
\hline pT3 & 89 & 36 & 40.4 & 28 & 31.5 & 25 & 28.1 & \\
\hline pT4 & 134 & 57 & 42.5 & 31 & 23.1 & 46 & 34.3 & \\
\hline \multicolumn{9}{|l|}{ pN-category } \\
\hline pNO & 212 & 82 & 38.7 & 52 & 24.5 & 78 & 36.8 & $N S^{b}$ \\
\hline pN0 (SN) & 118 & 34 & 28.8 & 37 & 31.4 & 47 & 39.8 & \\
\hline pN1 & 510 & 206 & 40.4 & 122 & 23.9 & 182 & 35.7 & \\
\hline $\mathrm{pN} 2 / 3$ & 149 & 56 & 37.6 & 38 & 25.5 & 55 & 36.9 & \\
\hline \multicolumn{9}{|l|}{ Menopausal status } \\
\hline Premenopausal & 261 & 99 & 37.9 & 67 & 25.7 & 95 & 36.4 & $\mathrm{NS}^{\mathrm{c}}$ \\
\hline Postmenopausal & 905 & 332 & 36.7 & 218 & 24.1 & 355 & 39.2 & \\
\hline \multicolumn{9}{|l|}{ Estrogen receptor status } \\
\hline Negative & 245 & 164 & 66.9 & 40 & 16.3 & 41 & 16.7 & $<0.0001^{\mathrm{b}}$ \\
\hline Positive & 1,105 & 330 & 29.9 & 293 & 26.5 & 482 & 43.6 & \\
\hline \multicolumn{9}{|l|}{ HER2 gene amplification } \\
\hline Not amplified & 1,152 & 404 & 35.1 & 283 & 24.6 & 465 & 40.4 & $<0.0001^{\mathrm{c}}$ \\
\hline Amplified & 169 & 81 & 47.9 & 39 & 23.1 & 49 & 29 & \\
\hline \multicolumn{9}{|l|}{ EGFR expression } \\
\hline Negative & 1,061 & 346 & 32.6 & 263 & 24.8 & 452 & 42.6 & $<0.0001^{\mathrm{b}}$ \\
\hline Positive & 275 & 139 & 50.5 & 63 & 22.9 & 73 & 26.5 & \\
\hline
\end{tabular}

node metastases, 3/78 (4\%) recurrences, and in 4/65 (6\%) distant metastases.

NY-BR-1 protein expression in recurrences and metastases

The NY-BR-1 expression in lymph node metastases $(60.2 \%)$ was comparable to that in primary tumors $(63.5 \%)$ but decreased to 52.2 and $49.4 \%$ in distant metastases and recurrences, respectively (Table 1). The lower NY-BR-1 expression in metastases than in primary lesions prompted us to compare NY-BR-1 expression in individual primary tumors and their corresponding metastases. A significant correlation was found between NY-BR-1 expression in primary tumors and their corresponding lymph node metastases $(P<0.0001)$ as well as in primary tumors and their corresponding distant metastases and recurrences $(P=0.0002,0.0045$, respectively). Fifteen out of $17(88 \%)$ NY-BR-1 weakly and strongly positive primary tumours expressed NY-BR-1 in their corresponding recurrence, whereas two initially positive tumours were negative in recurrences. Ten out of 14 (71\%) NY-BR-1 weakly and strongly positive primary tumours expressed also NY-BR-1 in their corresponding distant metastasis (data not shown). Comparison of immunohistochemical reactivity of NY-BR1 (as described in "Materials and methods") between primary tumors and lymph node metastasis $(n=406)$ revealed no statistical differences $(P=0.10)$. The immunohistochemical reactivity of NY-BR-1 was decreased in distant metastases $(n=36)$; this difference, however, did not reach the level of statistical significance. In contrast, the immunohistochemical reactivity of NY-BR-1 was significantly decreased in recurrences when compared to their primary counterparts $(P=0.019)$.

NY-BR-1 expression and patient prognosis

Preliminary data evaluated the quality of our survival data by correlating survival analyses with established prognostic factors. Low tumor grade, small tumor size, negative lymph node status, positive ER-status as well as negative EGFR 
Table 3 NY-BR-1 and HLA class I expression in primary tumors, recurrences, lymph node and distant metastases

\begin{tabular}{|c|c|c|c|c|c|c|c|}
\hline \multicolumn{8}{|c|}{ NY-BR-1 expression } \\
\hline & \multirow[t]{2}{*}{$n$} & \multicolumn{2}{|c|}{ Negative } & \multicolumn{2}{|c|}{ Weakly positive } & \multicolumn{2}{|c|}{ Strongly positive } \\
\hline & & $n$ & Percent & $n$ & Percent & $n$ & Percent \\
\hline \multicolumn{8}{|c|}{ (a) HLA class I expression in primary tumors } \\
\hline Negative & 408 & 163 & 40 & 81 & 19.9 & 164 & 40.2 \\
\hline Heterogeneous & 365 & 127 & 34.8 & 80 & 21.9 & 158 & 43.3 \\
\hline Positive & 548 & 199 & 36.3 & 151 & 27.6 & 198 & 36.1 \\
\hline \multicolumn{8}{|c|}{ (b) HLA class I expression in reccurrences } \\
\hline Negative & 33 & 15 & 45.5 & 8 & 24.2 & 10 & 30.3 \\
\hline Heterogeneous & 16 & 8 & 50 & 3 & 18.8 & 5 & 31.3 \\
\hline Positive & 29 & 17 & 58.6 & 9 & 31 & 3 & 10.3 \\
\hline \multicolumn{8}{|c|}{ (c) HLA class I expression in lymph node metastases } \\
\hline Negative & 192 & 72 & 37.5 & 38 & 19.8 & 82 & 42.7 \\
\hline Heterogeneous & 138 & 50 & 36.2 & 33 & 23.9 & 55 & 39.9 \\
\hline Positive & 140 & 65 & 46.4 & 30 & 21.4 & 45 & 32.1 \\
\hline \multicolumn{8}{|c|}{ (d) HLA class I expression in distant metastases } \\
\hline Negative & 33 & 17 & 51.5 & 8 & 24.2 & 8 & 24.2 \\
\hline Heterogeneous & 20 & 8 & 40 & 5 & 25 & 7 & 35 \\
\hline Positive & 12 & 7 & 58.3 & 1 & 8.3 & 4 & 33.3 \\
\hline
\end{tabular}

and HER2-status were found to be highly significant associated with improved clinical outcome in our patients $(P<0.0001$, each; data partly shown in Fig. 2 and Table 4).

Patients with strong NY-BR-1 expression had a better prognosis than patients with NY-BR-1 negative tumors ( $P=0.008$; data not shown). The 5-year overall survival for patients with strong expression was $83 \%$ compared to $74 \%$ for patients with NY-BR-1 negative tumors. Interestingly, when all patients were considered, those with weak NYBR-1 expression in their tumors resembled those with strong NY-BR-1 expression in the favorable clinical course of the disease. However, when only pre-menopausal patients were considered, those with weak NY-BR-1 expression in their tumors resembled those with NY-BR-1 negative tumor in the poor outcome of the disease (Fig. 2). An additional analysis showed that this effect was due to an increased number of high grade tumors with weak NY-BR1 positivity in the pre-menopausal group.

Multivariate analysis confirmed pT category, lymph node status, tumor grade and EGFR as well as HER2 genestatus to be independent prognostic markers (Table 4). NYBR-1 expression and ER-status are both linked to grading and were therefore not independent prognostic variables. HLA class I expression was not associated with patient outcome in uni- and multivariate analyses.

\section{Effect of chemotherapy on NY-BR-1 expression}

To test whether chemotherapy could have an effect on NYBR-1 expression, we analyzed 43-paired biopsies taken from patients just before starting neoadjuvant chemotherapy and 6 months after having received neoadjuvant chemotherapy. Seventeen patients received anthracyclines, two taxane and seventeen anthracyclines combined with taxanes. The type of chemotherapy could not be determined for the remaining seven patients. NY-BR-1 expression by IHC in paired biopsies was categorized into negative, weakly and strongly positive (as described in "Materials and methods"). The staining pattern of the biopsies obtained before and after therapy was not different in 40 (93\%) of the 43 patients investigated $(\kappa=0.89, P<0.0001$; 95\% CI 81-99\%). In this group, nine tumors were negative for NY-BR-1, 16 tumors expressed weakly and 15 strongly NY-BR-1. However, NY-BR-1 could not be detected in the biopsies obtained from two $(4 \%)$ patients after chemotherapy with initially weakly and strongly positive tumors. The latter two patients had both received anthracycline-containing chemotherapies. Initial absence of NY-BR-1 expression was observed in one patient $(2 \%)$, showing weak levels of NY-BR-1 in the tumor cells after chemotherapy. The type of chemotherapy could not be determined for this patient.

\section{Discussion}

In agreement with our recent immunohistochemical results obtained from 124 breast carcinoma lesions [12], the present study of more than 2,000 breast carcinoma lesions with a recently developed $\mathrm{mAb}$ has shown that NY-BR-1 is a breast epithelium differentiation antigen. Its expression is 


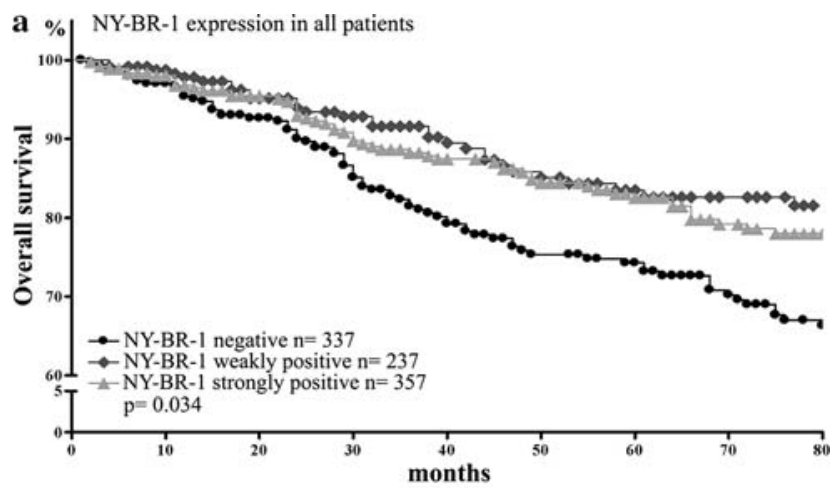

b $\%$ NY-BR-1 expression in premenopausal patients
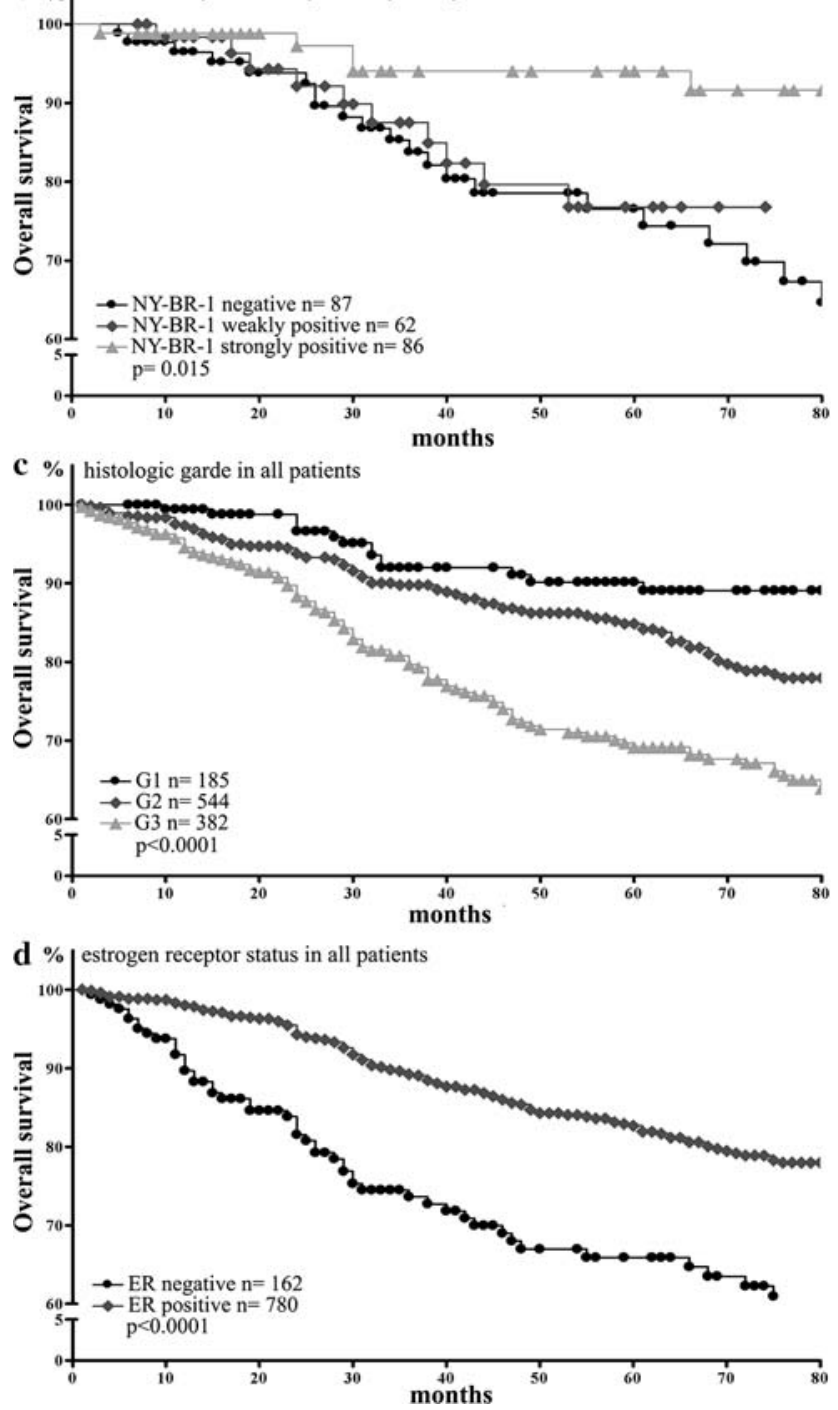

Fig. 2 Overall survival analysis according to NY-BR-1 expression in all patients (a), in premenopausal patients (b), and in all patients according to differentiation grade (c) and estrogen receptor $(E R)$ status (d)

associated with tumor differentiation, HER2-gene, EGFR and ER-status of breast cancer and prognosis. On large sections, we have observed that NY-BR-1 expression is heterogeneous in primary tumors [12]. Despite this intratumoral heterogeneity, the frequency of NY-BR-1 positive primary tumors on TMAs was comparable to that derived from large tissue section analysis. This indicates that the microarrray approach is suitable even when analyzing the expression of markers with a heterogeneous expression, because the large numbers of tumors compensate the small size of tissue samples [20, 21]. Due to its HLA class I coexpression and its stable expression in distant metastases NY-BR-1 appears to be a promising target for T-cell-based immunotherapy and vaccination strategies.

The present study has shown that multivariate analysis did not identify NY-BR-1 expression as an independent prognostic marker. In univariate analysis, strong NY-BR-1 expression is associated with a good prognosis due to the strong association of NY-BR-1 expression with tumor grade. Weak NY-BR-1 expression is associated with poor prognosis in pre-menopausal but not in postmenopausal patients. This finding can be explained by the larger number of high grade tumors in the group of pre-menopausal patients.

Like several other differentiation antigens, NY-BR-1 has a lower expression in metastatic lesions, because tumors of higher histological grade are more likely to metastasize. Specifically NY-BR-1 expression decreases in the following order: carcinoma in situ (CIS) $>$ invasive carcinoma $>$ lymph node metastasis $>$ distant metastasis. However, analysis of autologous primary and metastatic lesions of a particular patient showed a high concordance in NY-BR-1 expression in primary tumors and the corresponding lymph node metastases. Even distant metastases were found not to differ significantly from autologous primary tumors in NY-BR-1 expression level. Taken together, these results suggest that NY-BR-1 expression remains stable during disease progression. This finding is of particular interest, since therapeutic decisions in metastatic cancer are often based on the presence of biomarkers assessed solely in the primary tumor as it is almost always the case for the HER2-gene-status [17, 21, 22].

Cancer immunotherapy is potentially applied as an additional therapeutic strategy in combination with conventional chemo- or endocrine therapy. Therefore, knowledge about the relation of NY-BR-1 to other established therapeutic target antigens is crucial. According to our data, NYBR-1 is present in a large number of tumors without EGFR/ HER2 expression, suggesting that NY-BR-1 immunotherapy is a potential treatment option for patients with such tumors.

Breast cancer treatment largely depends on the hormone receptor status. Interestingly, NY-BR-1 expression was not associated to menopausal status, suggesting that estrogen levels might not influence NY-BR-1 expression to a large extent. However, the significant reduction of NY-BR-1 expression in local recurrences, especially when only 
Table 4 Univariate and multivariate analyzes including $\mathrm{pT}$ and $\mathrm{pN}$-category, histologic grade, $H E R 2$ gene-status, EGFR, estrogen receptor, NY-BR-1 and HLA class I

NS not significant

${ }^{a}$ increase of hazard for one stage (pT, pN, histologic grade) or negative to positive (EGFR, $H E R 2$-status, estrogen receptor, NY-BR-1, HLA class I) for overall survival

\begin{tabular}{|c|c|c|c|c|c|c|}
\hline \multirow[t]{2}{*}{ Variables } & \multicolumn{3}{|c|}{ Univariate } & \multicolumn{3}{|c|}{ multivariate } \\
\hline & $n$ & $\begin{array}{l}\text { Hazard ratio } \\
(95 \% \mathrm{CI})^{\mathrm{a}}\end{array}$ & $P$ value & $n$ & $\begin{array}{l}\text { Hazard ratio } \\
(95 \% \mathrm{CI})^{\mathrm{a}}\end{array}$ & $P$ value \\
\hline pT & 1,033 & $1.72(1.54-1.92)$ & $<0.0001$ & 728 & $1.78(1.53-2.06)$ & $<0.0001$ \\
\hline $\mathrm{pN}$ & 740 & $1.61(1.36-1.90)$ & $<0.0001$ & 728 & $1.38(1.15-1.65)$ & 0.0005 \\
\hline Histologic grade & 1,023 & $1.62(1.34-1.96)$ & $<0.0001$ & 728 & $1.46(1.13-1.88)$ & 0.003 \\
\hline EGFR & 1,038 & $1.45(1.12-1.87)$ & 0.005 & 728 & $1.91(1.39-2.61)$ & 0.0001 \\
\hline$H E R 2$ gene-status & 1,038 & $1.90(1.41-2.57)$ & 0.0001 & 728 & $1.59(1.12-2.24)$ & 0.009 \\
\hline Estrogen receptor & 1,038 & $0.55(0.42-0.72)$ & 0.0001 & 728 & - & NS \\
\hline NY-BR-1 & 1,038 & $0.74(0.59-0.96)$ & 0.015 & 728 & - & NS \\
\hline HLA class I & 1,038 & - & NS & 728 & - & NS \\
\hline
\end{tabular}

ER-positive, tamoxifen-treated patients were considered, is compatible with the possibility that estrogen antagonists might negatively affect NY-BR-1 expression (Theurillat et al., submitted). This possibility should be taken into account when evaluating NY-BR-1 as a target for immunotherapy.

An increasing body of evidence suggests that modification of the EGFR/HER2 signaling pathway by targeted cancer drugs offers novel treatment options for breast cancer. Trastuzumab against HER2 has been shown to be effective as an adjuvant therapy in combination with chemotherapy $[1,2]$. Recent clinical observations suggested that EGFRsignaling-inhibitors might restore anti-estrogene-sensitivity after tamoxifen-acquired resistance [23, 24]. Our previous study comprising a limited number of breast cancer lesions $(n=124)$ revealed a weak linear correlation between NY-BR-1 expression and HER2-amplication [12]. Sixty-nine percent of the primary breast cancer coexpressed NY-BR-1. In contrast, in the present study we found an inverse correlation between HER2/EGFR expression and NY-BR-1. This discrepancy is due to the fact that the former study included a much higher percentage of poorly differentiated, HER2-amplified tumors (percentage of HER2-amplification 57 vs. 14\%). The much higher number of tumors and a prevalence of HER2-amplification, which is comparable to the literature [25] indicate that the present results are more informative. Despite a weak inverse correlation, we found in the present study a coexpression of NY-BR-1 in about $50 \%$ of HER2-amplified as well as in EGFR positive carcinomas. Therefore, active specific immunotherapy targeting NY-BR-1 could be theoretically considered in combination to other targeted cancer drugs as an early, possibly adjuvant treatment option.

Currently, active specific immunotherapy is mostly applied in patients with metastatic disease, who have already received chemotherapy in an adjuvant or rarely in a neoadjuvant setting. Therefore it is important to know whether chemotherapy could influence NY-BR-1 expression. Our data indicate that neoadjuvant chemotherapy may have no effect on NY-BR-1 expression, suggesting that active specific immunotherapy targeting NY-BR-1 can be considered even in patients who have been treated with several chemotherapy cycles.

Successful implementation of T-cell-based immunotherapy of malignant diseases requires that HLA class I antigens are expressed on tumor cells and that they present tumor antigen derived peptides HLA class I antigen restricted, tumor antigen-specific cytotoxic T-cells [13, 14]. However, abnormalities in HLA class I antigen expression and/or function and/or in antigen-processing machinery are frequently found in human malignancies [26, 27]. Therefore knowledge about NY-BR-1 and HLA class I antigen co-expression are crucial, if T-cell based immunotherapy targeting NY-BR-1 is considered. In $15 \%$ of primary breast carcinoma, a strong coexpression of both antigens was observed. Although this frequency of HLA class I/NY-BR-1 co-expression in primary tumors is promising for T-cell-based immunotherapy, clinical trials will be primarily implemented in patients with metastatic disease. In distant metastases, NY-BR-1 and HLA class I was co-expressed to some extent in $26 \%$ of the cases-however, we observed only in $6 \%$ a strong co-expression of both antigens. The decreased level of coexpression was mainly due to a decrease of HLA class I expression observed during metastatic spread. Surprisingly, uni- and multivariate analysis did not show an association of HLA class I expression with prognosis in our patient cohort. This finding is in contrast to findings of Madjd et al. [28] recent results. The latter investigators identified HLA class I antigen loss as an independent marker of good prognosis. The discrepancy between Madjd et al. results and our own are not likely to reflect differences in the methodology used since in both studies TMA and mAb HC10 were utilized. A more likely explanation for the conflicting results is represented by differences in the characteristics of the patient populations investigated in the two studies.

In summary, we have demonstrated that NY-BR-1 meets most, if not all the criteria required to be utilized as a target for T-cell-based immunotherapy. 
Acknowledgments The skillful technical assistance of S. Bingge, and S. Behnke is greatly appreciated. The authors wish to thank N. Wey for his help in preparing the photographical illustrations and M. Aerne for supporting database programming. This study was partly supported by grants from LICR/CRI (Cancer Vaccine Collaborative, Cancer Antigen Discovery Collaborative), Claudia von Schilling Foundation for Breast Cancer Research, UBS AG Switzerland (made possible by an anonymous donor), Stiftung für die Forschung in der Onkologie and by PHS grants R01CA 67108 and R01CA 113861 awarded by the National Cancer Institute, DHHS. J. -Ph. Theurillat is supported by a grant form the "Gertrud-Hagmann-Stiftung für Malignomforschung". The authors do not have potential financial or personal conflicts of interest.

\section{References}

1. Piccart-Gebhart MJ, Procter M, Leyland-Jones B, Goldhirsch A, Untch M, Smith I, Gianni L, Baselga J, Bell R, Jackisch C, Cameron D, Dowsett M et al (2005) Trastuzumab after adjuvant chemotherapy in HER2-positive breast cancer. N Engl J Med 353:1659-1672

2. Romond EH, Perez EA, Bryant J, Suman VJ, Geyer CE Jr, Davidson NE, Tan-Chiu E, Martino S, Paik S, Kaufman PA, Swain SM, Pisansky TM et al (2005) Trastuzumab plus adjuvant chemotherapy for operable HER2-positive breast cancer. N Engl J Med 353:1673-1684

3. Jager D, Jager E, Knuth A (2001) Vaccination for malignant melanoma: recent developments. Oncology 60:1-7

4. Jager E, Ringhoffer M, Altmannsberger M, Arand M, Karbach J, Jager D, Oesch F, Knuth A (1997) Immunoselection in vivo: independent loss of MHC class I and melanocyte differentiation antigen expression in metastatic melanoma. Int J Cancer 71:142-147

5. Jager D, Jager E, Knuth A (2001) Immune responses to tumour antigens: implications for antigen specific immunotherapy of cancer. J Clin Pathol 54:669-674

6. Li G, Miles A, Line A, Rees RC (2004) Identification of tumour antigens by serological analysis of cDNA expression cloning. Cancer Immunol Immunother 53:139-143

7. Zendman AJ, Ruiter DJ, Van Muijen GN (2003) Cancer/testisassociated genes: identification, expression profile, and putative function. J Cell Physiol 194:272-288

8. Jager D, Taverna C, Zippelius A, Knuth A (2004) Identification of tumor antigens as potential target antigens for immunotherapy by serological expression cloning. Cancer Immunol Immunother 53:144-147

9. Jager D, Stockert E, Gure AO, Scanlan MJ, Karbach J, Jager E, Knuth A, Old LJ, Chen YT (2001) Identification of a tissue-specific putative transcription factor in breast tissue by serological screening of a breast cancer library. Cancer Res 61:2055-2061

10. Jager D, Karbach J, Pauligk C, Seil I, Frei C, Chen YT, Old LJ, Knuth A, Jager E (2005) Humoral and cellular immune response against the breast cancer antigen NY-BR-1: definition of two HLA-A2 restricted peptide epitopes. Cancer Immun 5:11

11. Wang W, Epler J, Salazar LG, Riddell SR (2006) Recognition of breast cancer cells by CD8+ cytotoxic T-cell clones specific for NY-BR-1. Cancer Res 66:6826-6833

12. Varga Z, Theurillat JP, Filonenko V, Sasse B, Odermatt B, Jungbluth AA, Chen YT, Old LJ, Knuth A, Jager D, Moch H (2006) Preferential nuclear and cytoplasmic NY-BR-1 protein expression in primary breast cancer and lymph node metastases. Clin Cancer Res 12:2745-2751

13. van Endert PM (1999) Genes regulating MHC class I processing of antigen. Curr Opin Immunol 11:82-88

14. Jager E, Jager D, Knuth A (2002) Clinical cancer vaccine trials. Curr Opin Immunol 14:178-182

15. Kononen J, Bubendorf L, Kallioniemi A, Barlund M, Schraml P, Leighton S, Torhorst J, Mihatsch MJ, Sauter G, Kallioniemi OP (1998) Tissue microarrays for high-throughput molecular profiling of tumor specimens. Nat Med 4:844-847

16. Ellis IO, Schnitt SJ, Sastre-Garau X, Bussolati G, Tavassoli FA, Eusebi V, Peterse JL, Mukai K, Tabar L, Jacquemier J, Cornelisse CJ, Sasco AJ et al (2003) Invasive breast carcinoma. In: Tavassoli FA, Devilee P (eds) Pathology and genetics tumours of the breast and female genital organs. IARC, Lyon, pp 13-48

17. Varga Z, Caduff R, Pestalozzi B (2005) Stability of the HER2 gene after primary chemotherapy in advanced breast cancer. Virchows Arch 446:136-141

18. Stam NJ, Spits H, Ploegh HL (1986) Monoclonal antibodies raised against denatured HLA-B locus heavy chains permit biochemical characterization of certain HLA-C locus products. J Immunol 137:2299-2306

19. Vitale M, Pelusi G, Taroni B, Gobbi G, Micheloni C, Rezzani R, Donato F, Wang X, Ferrone S (2005) HLA class I antigen downregulation in primary ovary carcinoma lesions: association with disease stage. Clin Cancer Res 11:67-72

20. Bubendorf L, Nocito A, Moch H, Sauter G (2001) Tissue microarray (TMA) technology: miniaturized pathology archives for high-throughput in situ studies. J Pathol 195:72-79

21. Simon R, Nocito A, Hubscher T, Bucher C, Torhorst J, Schraml P, Bubendorf L, Mihatsch MM, Moch H, Wilber K, Schotzau A, Kononen J et al (2001) Patterns of her-2/neu amplification and overexpression in primary and metastatic breast cancer. J Natl Cancer Inst 93:1141-1146

22. Dagrada GP, Mezzelani A, Alasio L, Ruggeri M, Romano R, Pierotti MA, Pilotti S (2003) HER-2/neu assessment in primary chemotherapy treated breast carcinoma: no evidence of gene profile changing. Breast Cancer Res Treat 80:207-214

23. Johnston SR, Hickish T, Ellis P, Houston S, Kelland L, Dowsett M, Salter J, Michiels B, Perez-Ruixo JJ, Palmer P, Howes A (2003) Phase II study of the efficacy and tolerability of two dosing regimens of the farnesyl transferase inhibitor, R115777, in advanced breast cancer. J Clin Oncol 21:2492-2499

24. Gee JM, Robertson JF, Gutteridge E, Ellis IO, Pinder SE, Rubini M, Nicolson RI (2005) Epidermal growth factor receptor/HER2/ insulin-like growth factor receptor signalling and oestrogen receptor activity in clinical breast cancer. Endocr Relat Cancer 12:99111

25. Sauer T, Wiedswang G, Boudjema G, Christensen H, Karesen R (2003) Assessment of HER-2/neu overexpression and/or gene amplification in breast carcinomas: should in situ hybridization be the method of choice? APMIS 111:444-450

26. Seliger B, Maeurer MJ, Ferrone S (2000) Antigen-processing machinery breakdown and tumor growth. Immunol Today 21:455-64

27. Hicklin DJ, Marincola FM, Ferrone S (1999) HLA class I antigen downregulation in human cancers: T-cell immunotherapy revives an old story. Mol Med Today 5:178-186

28. Madjd Z, Spendlove I, Pinder SE, Ellis IO, Durrant LG (2005) Total loss of MHC class I is an independent indicator of good prognosis in breast cancer. Int J Cancer 117:248-255 